\title{
Spatio-temporal analysis of place-naming dynamics in former West Prussia from 1772 to 2020
}

\author{
I. Doroshenko ${ }^{\mathrm{a}, *}$, F. Harvey ${ }^{\mathrm{b}}$ \\ ${ }^{a}$ Department of Cartography and Visual Communication, Leibniz Institute for Regional Geography, I_Doroshenko@leibniz-ifl.de \\ ${ }^{b}$ Department of Cartography and Visual Communication, Leibniz Institute for Regional Geography, f_harvey@leibniz-ifl.de \\ * Corresponding author
}

Keywords: Place-name research, Digital Humanities, Levenshtein distance

\begin{abstract}
:
Research into place-name changes has become important for interdisciplinary fields including the Digital Humanities. The quantification of toponym changes is not as trivial as it often seems at first glance. The data usually used to detect place-name changes are heterogeneous rather than just numerical due to the multitemporal and often multilingual sources.

This work presents a proposed framework to quantify place-name changes within a study area including former West Prussia (today's Poland), from 1772 to 2020. To achieve this goal, a quantitative analysis of 134 historical and modern data sources was conducted and almost 10,000 names for 73 places were investigated. First, the metadata infrastructure was created to capture the data sources with their attributes. Second, based on the metadata infrastructure, the spatiotemporal place-name database was constructed to collect place-names with their spatial and temporal characteristics. Third, the place-names were pre-processed, cleaned, and normalized. Finally, the framework quantifies toponym discrepancies using a two-metrics-approach based on the Levenshtein distance function. The first of the two metrics provides an understanding of how names from a particular historical period differ from the modern ones and the second metric offers insight into the variance of historical names. The workflow of the framework can be seen in the figure 1 .
\end{abstract}

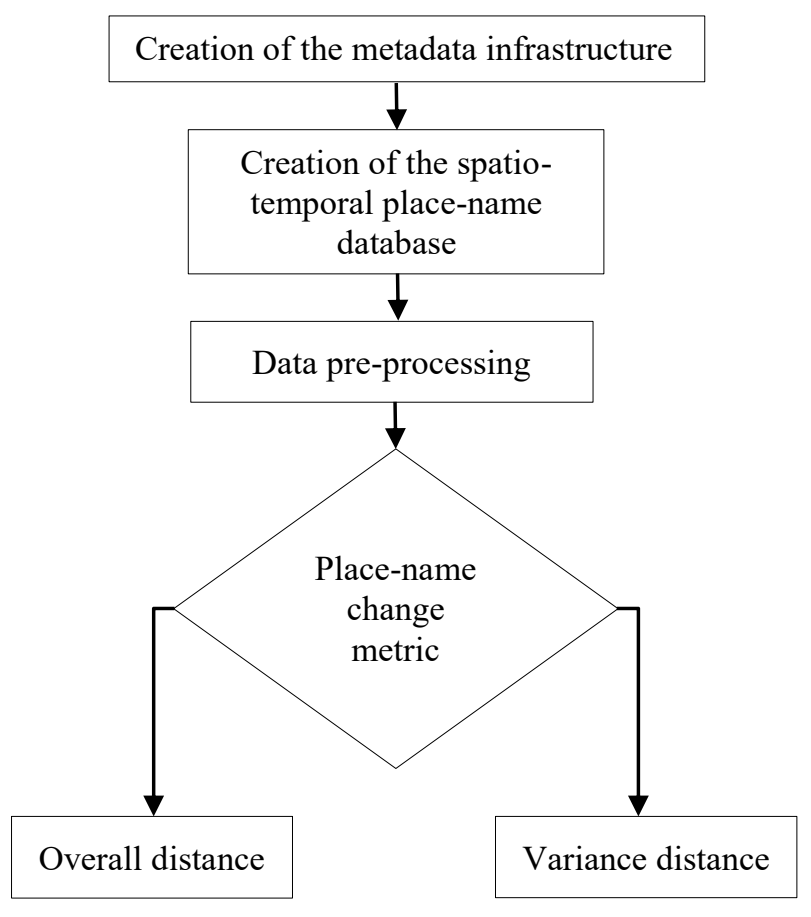

Figure 1. Workflow of a proposed place-name research framework

The analysis showed that both metrics do not have a stable linear dependency on each other, but follow specific spatial and temporal patterns which can be traced to the historical, linguistic, and ethnic characteristics of the region. This framework can be considered a basis for further quantitative and qualitative toponym research. Further modification and refinement of the techniques to track place-name changes over space and time will provide a valuable contribution to historical cartographic and toponym research. 